\title{
Fluctuations near the deconfinement phase transition boundary
}

\author{
I.N. Mishustin \\ Frankfurt Institute for Advanced Studies, J.-W. Goethe University, Max von \\ Laue Str. 1, D-60438 Frankfurt am Main, Germany \\ The Kurchatov Institute, Russian Research Center, 123182 Moscow, Russia
}

\begin{abstract}
In this talk I discuss how a first order phase transition may proceed in rapidly expanding partonic matter produced in a relativistic heavy-ion collision. The resulting picture is that a strong collective flow of matter will lead to the fragmentation of a metastable phase into droplets. If the transition from quark-gluon plasma to hadron gas is of the first order, it will manifest itself by strong nonstatistical fluctuations in observable hadron distributions. I discuss shortly existing experimental data on the multiplicity fluctuations.
\end{abstract}

\section{Introduction}

A general goal of present and future experiments with heavy-ion beams is to study the properties of strongly interacting matter away from the nuclear ground state. The main interest is focussed on searching for possible phase transitions. Several phase transitions are predicted in different domains of temperature $T$ and baryon density $\rho_{B}$. As well known, strongly interacting matter has at least one multi-baryon bound state at $\rho_{B}=\rho_{0} \approx 0.16$ $\mathrm{fm}^{-3}$ corresponding to normal nuclei. It follows from the very existence of this bound state that there should be a first order phase transition of the liquid-gas type in normal nuclear matter at subsaturation densities, $\rho_{B}<\rho_{0}$, and low temperatures, $T \leq 10 \mathrm{MeV}$. This phase transition manifests itself in a remarkable phenomenon known as nuclear multifragmentation.

The situation at high $T$ and nonzero baryon chemical potential $\mu_{B}\left(\rho_{B}>0\right)$ is not so clear, although everybody is sure that the deconfinement and chiral transitions should occur somewhere. Reliable lattice calculations exist only for $\mu=0$ i.e. $\rho_{B}=0$ where they predict a smooth deconfinement transition (crossover) at $T \approx 170 \mathrm{MeV}$. As model calculations show, the phase diagram in the $\left(T, \mu_{B}\right)$ plane may contain a first order transition line (below called the critical line) which ends at a (tri)critical point [1, 2, 3]. Possible signatures of this point in heavy-ion collisions are discussed in ref. [4. However, it is unclear at present whether critical fluctuations associated with the second order phase transition can develop in a rapidly expanding system produced in a relativistic heavy-ion collison because of the critical slowing down effect [5. In my opinion, more promissing strategy would be to search for a first order phase transition which should have much more spectacular manifestations 6] discussed below. It is interesting to note that, under certain non-equilibrium conditions, a first order transition is also predicted for symmetric quark-antiquark matter with zero net baryon density [7.

A striking feature of central heavy-ion collisions at high energies, confirmed in many experiments (see e.g. [8, 9]), is a very strong collective expansion of matter at later stages of the reaction. This process looks like an explosion with the matter flow velocities comparable with the speed of light. The applicability of equilibrium concepts for describing 
phase transitions under such conditions becomes questionable and one should expect strong non-equilibrium effects [10, 11, 12. In this talk I demonstrate that non-equilibrium phase transitions in rapidly expanding matter can lead to interesting phenomena which, in a certain sense, are even easier to observe.

\section{Effective thermodynamic potential}

To make the discussion more concrete, in this talk I adopt a picture of the chiral phase transition predicted by the linear sigma-model with constituent quarks [3, 13]. Then the mean chiral field $\Phi=(\sigma, \pi)$ serves as an order parameter. The constituent quark mass is generated by interaction with the sigma field, $m=g \sigma$, where $g$ is a corresponding coupling constant. The effective thermodynamic potential $\Omega(\Phi ; T, \mu)$ depends, besides $\Phi$, on temperature $T$ and quark chemical potential $\mu=\mu_{B} / 3$. This model respects chiral symmetry which is spontaneously broken in the vacuum, where the sigma field has a nonzero expectation value, $\langle\sigma\rangle=f_{\pi},\langle\pi\rangle=0$. It is important for our discussion below that the model predicts a phase diagram on the $(T, \mu)$ plane with a critical point at $(\mathrm{T}=100 \mathrm{Mev} . \mu=207 \mathrm{MeV})$ and a first order phase transition line at lower $\mathrm{T}$ and $\mu$. A schematic behaviour of $\Omega(T, \mu ; \Phi)$ as a function of the order parameter field $\sigma$ at $\pi=0$ is shown in Fig. 1. The minima of $\Omega$ determine the stable or metastable states of matter under the condition of thermodynamical equilibrium, where the pressure is $P=-\Omega_{\text {min }} / V$. The curves from bottom to top correspond to homogeneous matter at different quark chemical potentials and fixed temperature $T=0$. The dash-dotted curve corresponds to the first order phase transition point (two equal minima separated by a potential barrier). Two dashed curves show the thermodynamic potential at upper and lower spinodal points, where one of the minima disappears. The range of thermodynamic parameters where two phases, one stable and one metastable, may exist simulteneously is constrained by these two curves. The critical point would correspond to the situation when two minima fuse and the barrier disappears. This situation is illustrated in Fig. 2 showing the thermodynamic potential at different temperatures and fixed chemical potential $\mu_{c}=207 \mathrm{MeV}$. This model reveals a rather weak first order phase transition, although some other models [1, 2] predict a stronger transition. The discussion below is quite general.

One can plot a family of curves for the $(T, \mu)$ values corresponding to an isentropic expansion of matter. Qualitatively the potential curves look similar to the ones depicted in Fig. 1. Assume that at some early stage of the reaction the thermal equilibrium is established, and partonic matter is in a "high energy density" phase Q (lowest curve). This state corresponds to the absolute minimum of $\Omega$ with the order parameter close to zero, $\sigma \approx 0, \pi=0$, and chiral symmetry restored. Due to a very high internal pressure, Q matter will expand and cool down. At some stage a metastable minimum appears in $\Omega$ at a finite value of $\sigma$ corresponding to a "low energy density" phase $\mathrm{H}$ (lower dashed curve), in which chiral symmetry is spontaneously broken. At some later time, the critical line in the $(T, \mu)$ plane is crossed where the $\mathrm{Q}$ and $\mathrm{H}$ minima have equal depths, i.e. $P_{\mathrm{H}}=P_{\mathrm{Q}}$ (dot-dashed curve). At later times the $\mathrm{H}$ phase becomes more favorable, but the two phases are still separated by a potential barrier. At certain stage the minimum corresponding to the $\mathrm{Q}$ phase dissapears (upper dashed curve). The dashed curves separate the regions in the phase diagram where one of the phases is unstable (spinodal points). If the expansion of the $\mathrm{Q}$ phase continues until the barrier vanishes, the system will find itself in an absolutely unstable state at a maximum of the thermodynamic potential. Therefore, it will freely roll down into 


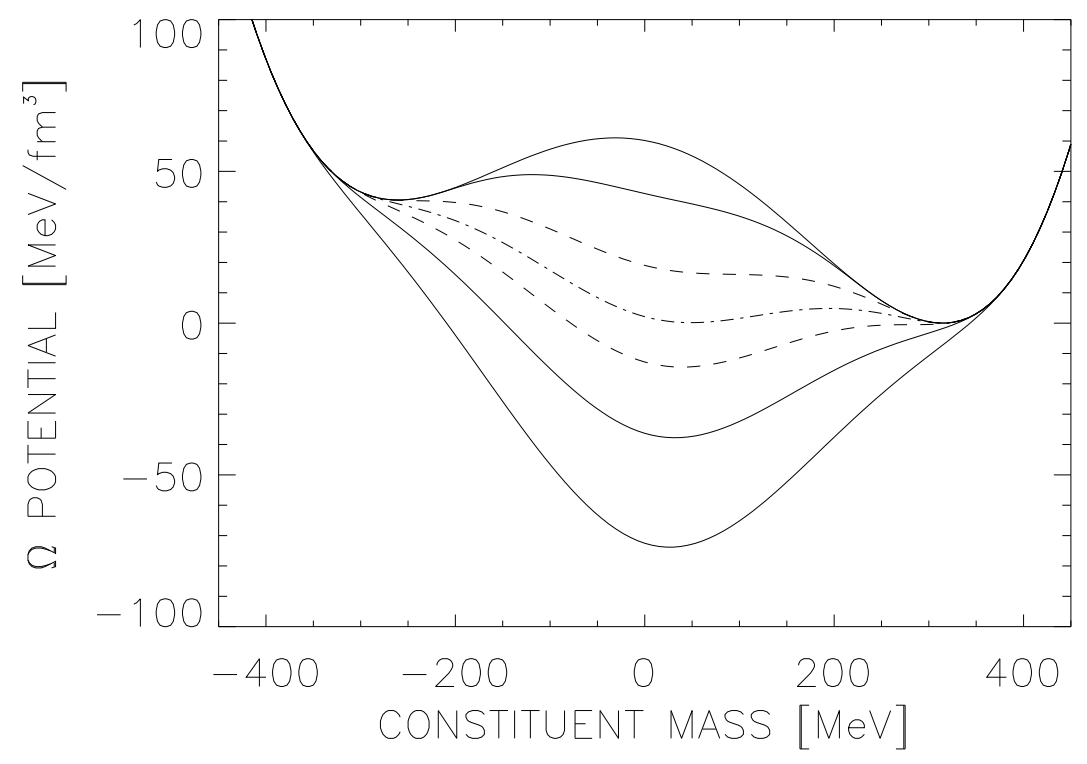

Figure 1: The thermodynamical potential $\Omega$ for the sigma model at $T=0$ and quark chemical potentials (starting from the top): $\mu=[0,225,279,306,322,345,375] \mathrm{MeV}$.

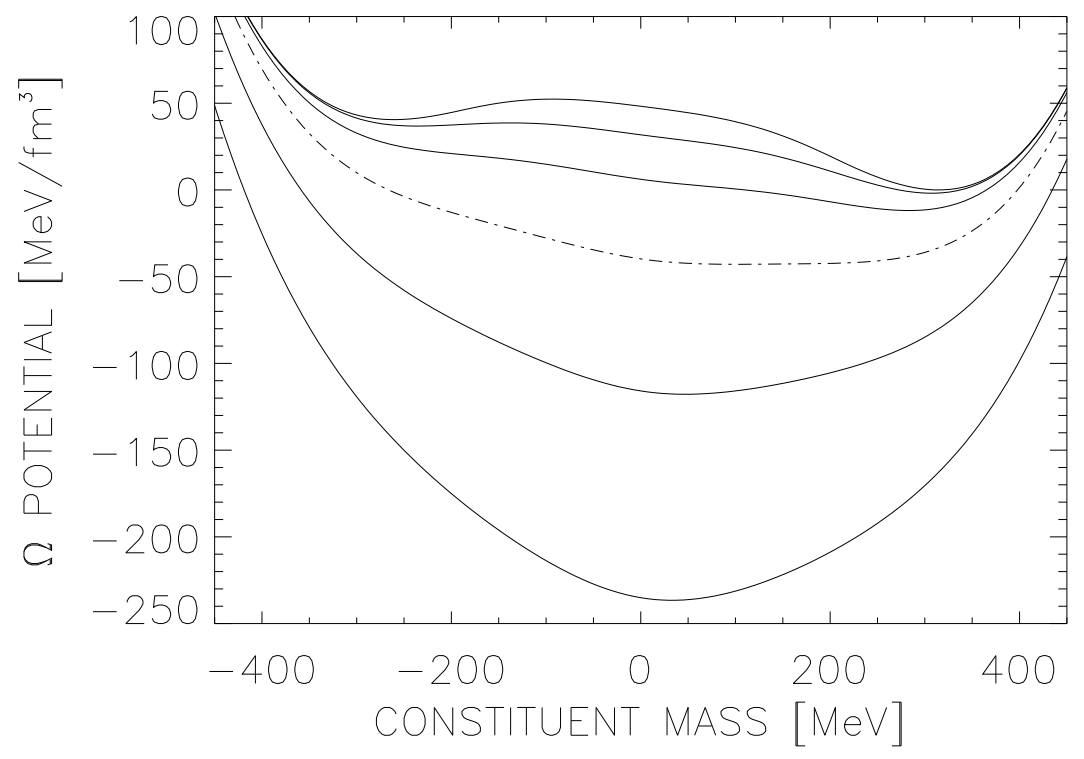

Figure 2: The thermodynamical potential $\Omega$ for the sigma model for the sigma model at $\mu$ fixed to $207 \mathrm{MeV}$ and temperatures (starting from the top): $T=[0,50,75,100,125,150]$ $\mathrm{MeV}$. 
the lower energy state corresponding to the H phase. This situation is known as spinodal instability. As shown e.g. in ref. [14, the characteristic time of the "rolling down" process is relatively short, of about $1 \mathrm{fm} / \mathrm{c}$.

As well known, a first order phase transition proceeds through the nucleation process. According to the standard theory of homogeneous nucleation [15], supercritical bubbles of the $\mathrm{H}$ phase can appear only below the critical line, when $P_{H}>P_{Q}$. Then the critical radius for growing bibbles is $R_{c}=2 \gamma /\left(P_{H}-P_{Q}\right)$, where $\gamma$ is the interface energy per unit area (surface tention). In rapidly expanding matter the nucleation picture might be very different. As argued in ref. [6], the phase separation in this case can begin as early as the metastable $\mathrm{H}$ state appears in the thermodynamic potential, and a stable interface between the two phases may exist. An appreciable amount of nucleation bubbles and even empty cavities may be created already above the critical line. They are stabilized by the collective expansion of matter.

The bubble formation and growth will also continue below the critical line. Previously formed bubbles will now grow faster due to increasing pressure difference, $P_{\mathrm{H}}-P_{\mathrm{Q}}>0$, between the two phases. It is most likely that the conversion of $\mathrm{Q}$ matter on the bubble boundary is not fast enough to saturate the $\mathrm{H}$ phase. Therefore, a fast expansion may lead to a deeper cooling of the $\mathrm{H}$ phase inside the bubbles compared to the surrounding $\mathrm{Q}$ matter. Strictly speaking, such a system cannot be characterized by the unique temperature. At some stage the $\mathrm{H}$ bubbles will percolate, and the topology of the system will change to isolated regions of the $\mathrm{Q}$ phase $(\mathrm{Q}$ droplets) surrounded by the undersaturated vapor of the H phase.

\section{$3 \quad$ Fragmentation of a metastable phase}

The characteristic droplet size can be estimated by applying the energy balance consideration, proposed by Grady [16, 17] in the study of dynamical fragmentation of fluids. The idea is that the fragmentation of expanding matter is a local process minimizing the sum of surface and kinetic (dilational) energies per fragment volume. As shown in ref. [21], this prescription works fairly well for the multifragmentation of expanding nuclear systems produced in intermediate-energy heavy-ion reactions, where the standard statistical approach fails.

Let us consider first an isotropically expanding system where the collective velocity field follows locally the Hubble law

$$
v(r)=H \cdot r,
$$

where $H$ is a Hubble constant. Since there is no preferred direction, the droplets of the $\mathrm{Q}$ phase will have a more or less spherical shape. The characteristic radius of the droplet, $R$, can be estimated as follows [16. The energy $\Delta E$ associated with a $\mathrm{Q}$ droplet embedded in a background of the $\mathrm{H}$ phase is represented as the sum of three terms,

$$
\Delta E=E_{\text {bulk }}+E_{\text {kin }}+E_{\text {sur }}
$$

The bulk term is simply equal to $\Delta \mathcal{E} V$ where $\Delta \mathcal{E}=\mathcal{E}_{Q}-\mathcal{E}_{H}$ is the energy density difference between the two bulk phases and $V \propto R^{3}$ is the volume of the droplet. The second term is the collective kinetic energy of the droplet expansion with respect to its center of mass,

$$
E_{\text {kin }}=\frac{1}{2} \int \mathcal{E} v^{2}(r) d V=\frac{3}{10} \Delta \mathcal{E} V H^{2} R^{2}
$$


The last term in eq. (2) is the interface energy, $E_{\text {sur }}=\gamma S$, which is parametrized in terms of the effective surface tension $\gamma$ and the surface area $S \propto R^{2}$. Grady's argument is that the redistribution of matter is a local process minimizing the energy per droplet volume, $\Delta E / V$. Then the bulk contribution does not depend on $R$, and the minimization condition constitutes the balance between the collective kinetic energy and interface energy. This leads to the optimum radius of a droplet

$$
R^{*}=\left(\frac{5 \gamma}{\Delta \mathcal{E} H^{2}}\right)^{1 / 3}
$$

It is worth noting that the collective kinetic energy acts here as an effective long-range potential similar to the Coulomb potential in nuclei.

At ultrarelativistic collision energies associated with RHIC and LHC experiments, the expansion of partonic matter will be very anisotropic with its strongest component along the beam direction [18. Clear indications of such an anisotropy are seen already at SPS energies (see [9]). It is natural to think that in this case the inhomogeneities associated with the phase transition will rearrange into pancake-like slabs of $\mathrm{Q}$ matter embedded in a dilute $\mathrm{H}$ phase. The characteristic width of the slab, $2 L$, can be estimated in a similar way and the resulting expression for $L^{*}$ differs from Eq. (4) only by a geometrical factor (3 instead of 5 in parentheses). Generally, the faster is the expansion, the smaller are the fractures. Of course, at a later time the $\mathrm{Q}$ droplets will further fragment in the transverse direction due to the standard nucleation process.

As Eq. (41) indicates, the droplet size depends strongly on $H$. When expansion is slow (small $H$ ) the droplets are big. Ultimately, the process may look like a fission of a cloud of plasma. But fast expansion should lead to very small droplets. This state of matter is very far from thermodynamical equilibrium, particularly because the $\mathrm{H}$ phase is very dilute. One can say that the metastable Q matter is torn apart by a mechanical strain associated with the collective expansion. This has a direct analogy with the fragmentation of pressurized fluids leaving nozzles. In a similar way, splashed water makes droplets which have nothing to do with the liquid-gas phase transition.

The driving force for expansion is the pressure gradient, $\nabla P=\equiv c_{s}^{2} \nabla \mathcal{E}$, which depends crucially on the sound velocity in the matter, $c_{s}$. Here we are interested in the expansion rate of the partonic phase which is not directly observable. In the vicinity of the phase transition, one may expect a "soft point" [19, 20] where the sound velocity is smallest and the ability of matter to generate the collective expansion is minimal. If the initial state of the $\mathrm{Q}$ phase is close to this point, its subsequent expansion will be slow. Accordingly, the droplets produced in this case will be big. When moving away from the soft point, one would see smaller and smaller droplets. For numerical estimates we choose two values of the Hubble constant: $H^{-1}=20 \mathrm{fm} / \mathrm{c}$ to represent the slow expansion from the soft point and $H^{-1}=6 \mathrm{fm} / \mathrm{c}$ for the fast expansion.

One should also specify two other parameters, $\gamma$ and $\Delta \mathcal{E}$. The surface tension $\gamma$ is a subject of debate at present. Lattice simulations indicate that at the critical point it could be as low as a few $\mathrm{MeV} / \mathrm{fm}^{2}$. However, for our non-equilibrium scenario, more appropriate values are closer to $10-20 \mathrm{MeV} / \mathrm{fm}^{2}$ which follow from effective chiral models. As a compromise, the value $\gamma=10 \mathrm{MeV} / \mathrm{fm}^{2}$ is used below. Bearing in mind that nucleons and heavy mesons are the smallest droplets of the $\mathrm{Q}$ phase, one can take $\Delta \mathcal{E}=0.5 \mathrm{GeV} / \mathrm{fm}^{3}$, i.e. the energy density inside the nucleon. Then one gets $R^{*}=3.4 \mathrm{fm}$ for $H^{-1}=20 \mathrm{fm} / \mathrm{c}$ and $R^{*}=1.5 \mathrm{fm}$ for $H^{-1}=6 \mathrm{fm} / \mathrm{c}$. As follows from eq. (4), for a spherical droplet $V \propto 1 / \Delta \mathcal{E}$, and in the first 
approximation its mass,

$$
M^{*} \approx \Delta \mathcal{E} V=\frac{20 \pi}{3} \frac{\gamma}{H^{2}},
$$

is independent of $\Delta \mathcal{E}$. For two values of $R^{*}$ given above the mass is $\sim 100 \mathrm{GeV}$ and $\sim 10$ $\mathrm{GeV}$, respectively. The pancake-like droplets could be heavier due to their larger transverse size. Using the minimum information principle one can show [17, 21] that the distribution of droplets should follow an exponential law, $\exp \left(-\frac{M}{M^{*}}\right)$. Thus, about $2 / 3$ of droplets have masses smaller than $M^{*}$, but with $1 \%$ probability one can find droplets as heavy as $5 M^{*}$.

\section{Observable manifestations of quark droplets}

After separation, the droplets recede from each other according to the Hubble law, like galaxies in expanding Universe. Therefore, their c.m. rapidities are in one-to-one correspondence with their spatial positions. One may expect that they are distributed more or less uniformly between the target and the projectile rapidities. On this late stage it is unlikely that the thermodynamical equilibrium is re-established between the $\mathrm{Q}$ and $\mathrm{H}$ phases or within the $\mathrm{H}$ phase alone. If this were to happen, the final $\mathrm{H}$ phase would be uniform, and thus there would be no traces of the droplet phase in the final state.

The final fate of individual droplets depends on their sizes and on details of the equation of state. Due to the additional Laplace pressure, $2 \gamma / R$, the residual expansion of individual droplets will slow down. The smaller droplets may even reverse their expansion and cooling to shrinking and reheating. Then, the conversion of $\mathrm{Q}$ matter into $\mathrm{H}$ phase may proceed through the formation of the imploding deflagration front [20, 22]. Bigger droplets may expand further until they enter the region of spinodal instability At this stage the difference between 1-st and 2-nd order phase transitions or a crossover is insignificant. Since the characteristic "rolling down" time is rather short, $\sim 1 \mathrm{fm} / \mathrm{c}$, the Q droplets will be rapidly converted into the non-equilibrium $\mathrm{H}$ phase. In refs. [10, 12] the evolution of individual droplets was studied numerically within a hydrodynamical approach including dynamical chiral fields (Chiral Fluid Dynamics). It has been demonstrated that the energy released at the spinodal decomposition can be transferred directly into the collective oscillations of the $(\sigma, \pi)$ fields which give rise to the soft pion radiation. One can also expect the formation of Disoriented Chiral Condensates (DCC) in the voids between the Q droplets.

An interesting possibility arises if the metastable Q phase has a point of zero pressure. In particular, this is the case for the MIT bag model equation of state at temperatures only slightly below $T_{c}$ [23]. In this case the droplets might be in mechanical equilibrium with the surrounding vacuum $\left(P_{H} \approx 0\right)$, like atomic nuclei or water droplets. The equilibrium condition is

$$
P_{Q}=\frac{\nu_{Q}}{2 \pi^{2}}\left[\frac{7 \pi^{4}}{180} T^{4}+\frac{\pi^{2}}{6} T^{2} \mu^{2}+\frac{1}{12} \mu^{4}\right]-B=\frac{2 \gamma}{R},
$$

where $\nu_{Q}=12$ is the degeneracy factor for massless $\mathrm{u}$ and $\mathrm{d}$ quarks (the gluon contribution is omitted here), and $B$ is a bag constant. The evolution is then governed by the evaporation of hadrons from the surface (see also the discussion in Ref. [24]). One can speculate about all kinds of exotic objects, like e.g. strangelets, glueballs, formed in this way. The possibility of forming "vacuum bubbles', i.e. regions with depleted quark and gluon condensates, was discussed in ref. [10]. All these interesting possibilities deserve further study and numerical simulations. 


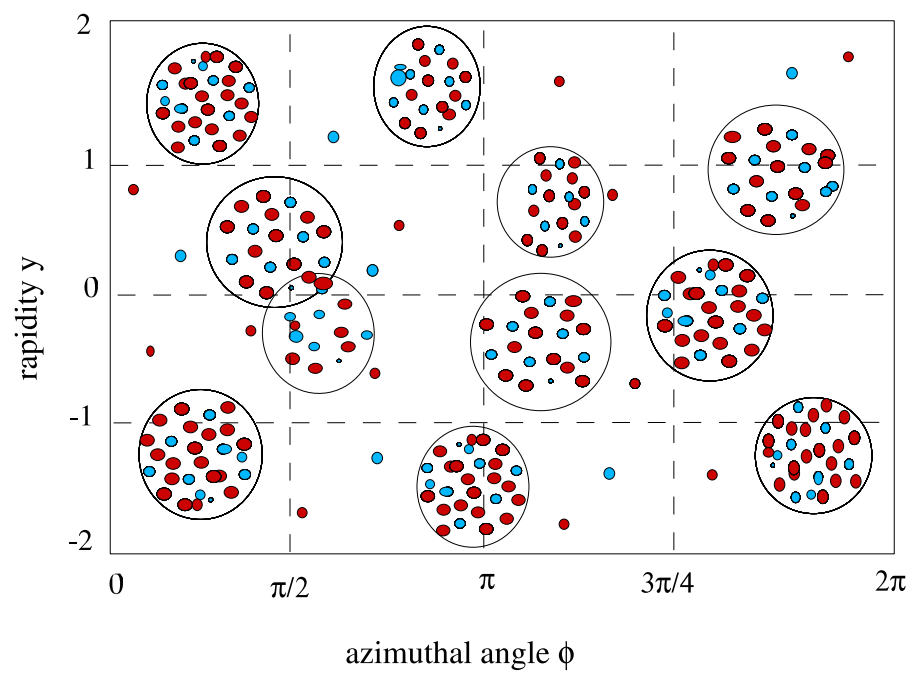

Figure 3: Schematic view of the momentum space distribution of secondary hadrons produced from an ensemble of droplets. Each droplet emtts hadrons (mostly pions) within a rapidity interval $\delta y \sim 1$ and azimuthal angle spreading of $\delta \phi \sim 1$.

After separation the QGP droplets recede from each other according to the global expansion, predominantly along the beam direction. Hence their center-of-mass rapidities $y_{i}$ are in one-to-one correspondence with their spatial positions. Presumably $y_{i}$ will be distributed more or less evenly between the target and projectile rapidities. Since rescatterings in the dilute $\mathrm{H}$ phase are rare, most hadrons produced from individual droplets will go directly into detectors. This may explain why freeze-out parameters extracted from the hadronic yields are always very close to the phase transition boundary [25].

In the droplet phase the mean number of produced hadrons in a given rapidity interval is

$$
\langle N\rangle=\sum_{i}^{N_{D}} \overline{n_{i}}=\langle n\rangle\left\langle N_{D}\right\rangle,
$$

where $\overline{n_{i}}$ is the mean multiplicity of hadrons emitted from a droplet $\mathrm{i},\langle n\rangle$ is the average multiplicity per droplet and $\left\langle N_{D}\right\rangle$ is the mean number of droplets produced in this interval. If droplets do not overlap in the rapidity space, each droplet will give a bump in the hadron rapidity distribution around its center-of-mass rapidity $y_{i}$ [14. In case of a Boltzmann spectrum the width of the bump will be $\delta \eta \sim \sqrt{T / m}$, where $T$ is the droplet temperature and $m$ is the particle mass. At $T \sim 100 \mathrm{MeV}$ this gives $\delta \eta \approx 0.8$ for pions and $\delta \eta \approx 0.3$ for nucleons. These spectra might be slightly modified by the residual expansion of droplets. Due to the radial expansion of the fireball the droplets should also be well separated in the azimuthal angle. The characteristic angular spreading of pions produced by an individual droplet is determined by the ratio of the thermal momentum of emitted pions to their mean transverse momentum, $\delta \phi \approx 3 T /\left\langle p_{\perp}\right\rangle \sim 1$. The resulting phase-space distribution of hadrons in a single event will be a superposition of contributions from different $\mathrm{Q}$ droplets superimposed on a more or less uniform background from the $\mathrm{H}$ phase. Such a distribution is shown schemmatically in Fig. 3. It is obvious that such inhomogeneities (clusterization) in the momentum space will reveal strong non-statistical fluctuations. The fluctuations will be more pronounced if primordial droplets are big, as expected in the vicinity of the soft 
point. If droplets as heavy as $100 \mathrm{GeV}$ are formed, each of them will emit up to $\sim 200$ pions within a narrow rapidity and angular intervals, $\delta \eta \sim 1, \delta \phi \sim 1$. If only a few droplets are produced in average per unit rapidity, $N_{D} \gtrsim 1$, they will be easily resolved and analyzed. On the other hand, the fluctuations will be suppressed by factor $\sqrt{N_{D}}$ if many small droplets shine in the same rapidity interval.

\section{Anomalous multiplicity fluctuations}

For our discussion below we consider a more general case of the droplet mass distribution when masses follow a gamma-distribution

$$
w_{k}(M)=\frac{b}{\Gamma(k)}(b M)^{k-1} \exp (-b M)
$$

which is normalized for $0 \leq M \leq \infty$. The mean mass and its standard deviation are expressed through the parameters $k$ and $b$ as

$$
<M>=\frac{k}{b}, \sigma_{M}=\frac{\sqrt{k}}{b}=\frac{<M>}{\sqrt{k}} .
$$

These expressions show that quantity $1 / \sqrt{k}$ gives the relative scale of fluctuations of $M$ around $\langle M\rangle$. It should be stressed that the gamma-distribution (8) drops at large $M$ less rapidly than a corresponding gaussian distribution.

One can easily calculate the combined multiplicity distribution produced by the ensemble of many droplets. Let us assume that the normalized mass distribution of droplets is $w_{k}(M)$ and that each droplet emits hadrons according to the Poisson law, $p_{\bar{n}}(n)$, with the mean multiplicity proportional to the droplet mass, $\bar{n}=M /\left\langle E_{\pi}\right\rangle$ (for pions $\left\langle E_{\pi}\right\rangle \approx 3 T \sim 0.5$ $\mathrm{GeV})$. Then the combined distribution is given by the convolution of the two,

$$
P_{k}(N)=\int_{0}^{M} d M w_{k}(M) p_{\bar{n}}(N) .
$$

For the gamma-distribution (8) one can perform explicit analytical calculations. It is remarkable that the resulting distribution is a famous Negative Binomial Distribution (NBD)

$$
P_{k}(N)=\frac{(N+k-1) !}{N !(k-1) !} \frac{\left(\frac{\langle N\rangle}{k}\right)^{N}}{\left(1+\frac{\langle N\rangle}{k}\right)^{N+k}} .
$$

In a limiting case of the exponential mass distribution $(\mathrm{k}=1)$ the combined distribution is simply given by

$$
P_{1}(N)=\frac{1}{\langle N\rangle}\left(\frac{\langle N\rangle}{1+\langle N\rangle}\right)^{N+1},
$$

where $\langle N\rangle=\langle\bar{n}\rangle=\alpha\langle M\rangle$ is the mean total multiplicity.

It is convenient to characterize the fluctuations by the scaled variance

$$
\omega_{N} \equiv \frac{\left\langle N^{2}\right\rangle-\langle N\rangle^{2}}{\langle N\rangle} .
$$


Its important preperty is that $\omega_{N}=1$ for the Poisson distribution, and therefore any deviation from unity will signal a non-statistical emission mechanism. For the NBD, eq. (??), one easily finds $\omega_{N}=1+\langle N\rangle / k$. As shown in ref. [26], for an ensemble of emitting sources (droplets) $\omega_{N}$ can be expressed in a simple form, $\omega_{N}=\omega_{n}+\langle n\rangle \omega_{D}$, where $\omega_{n}$ is an average multiplicity fluctuation in a single droplet, $\omega_{D}$ is the fluctuation in the droplet size distribution and $\langle n\rangle$ is the mean multiplicity from a single droplet. Since $\omega_{n}$ and $\omega_{D}$ are typically of order of unity, the fluctuations from the multi-droplet emission are enhanced by the factor $\langle n\rangle$. According to the picture of a first order phase transition advocated above, this enhancement factor could be as large as $10^{2}$. It is clear that the nontrivial structure of the hadronic spectra will be washed out to a great extent when averaging over many events. Therefore, more sophisticated methods of the event sample analysis should be applied. As demonstrated below, the simplest one is to search for non-statistical fluctuations in the hadron multiplicity distributions measured in a varied rapidity bin.

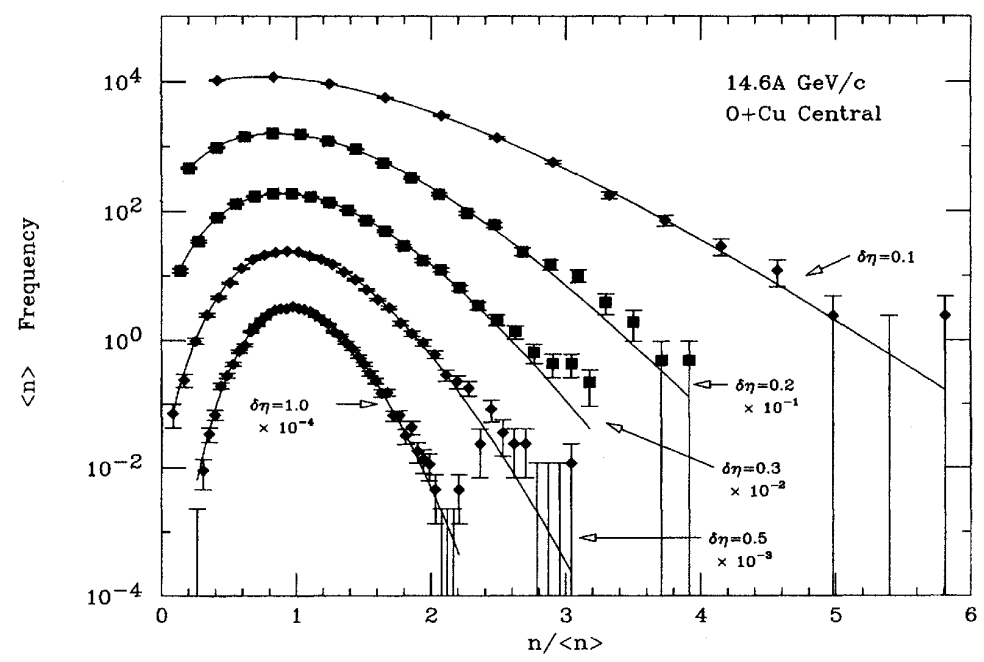

Figure 4: Event-by-event multiplicity distributions in $\delta \eta$ intervals $0.1,0.2,0.3,0.5,1.0$ measured by E-802 Collaboration for central ${ }^{16} \mathrm{O}+\mathrm{Cu}$ collisions at lab energy $14.6 \mathrm{AGeV}$ 27. The data for each interval are plotted as a function of $n /\langle n\rangle$ and scaled by $\langle n\rangle$, the mean multiplicity in the interval. Each successive distribytion has been normalized by the factor indicated in the figure. The shape evolvs from almost Gaussian $(\delta \eta=1.0)$ to nearly exponential $(\delta \eta=0.1)$.

The event-by-event multiplicity fluctuations observed so far in heavy-ion experiments do not reveal any anomalous enhancement. Fig. 4 shows an example of the multiplicity distributions in varying pseudorapidity intervals measured for $\mathrm{O}+\mathrm{Cu}$ central collisions at AGS [27. These distributions can very well be fitted by the NBD where parameters $\mathrm{k}(\delta \eta)$ and $\langle N(\delta \eta)\rangle$ follow a linear relationship with nonzero intercept $k(0)$. The data show an increase in scaled variances of about $15 \div 30 \%$ over 1 . Apparantly such moderate deviations can be explained by ordinary reasons, not related to a phase transition. An interseting observation has been made by the NA49 collaboration 28, which has found non-monotonic behaviour of $\omega_{N}$ as a function of the projectile participant number $N_{p}$. But in this case too the actual values of $\omega_{N}$ are only of about 2 , and most likely they can be explained by the fluctuations in the number of target participants [29]. 
It should be noted that the NBD fits were first used to describe the multiplicity distributions in high-energy $p p$ and $p \bar{p}$ collions (see e.g. ref. [30]). They are consistent with the so called KNO scaling [31].

\section{Conclusions}

- A first order phase transition in rapidly expanding matter should proceed through the nonequilibrium stage when a metastable phase splits into droplets whoose size is inversly proposrtional to the expansion rate. The primordial droplets should be biggest in the vicinity of a soft point when the expansion is slowest.

- Hadron emission from droplets of the quark-gluon plasma should lead to large nonstatistical fluctuations in their rapidity and azimuthal spectra, as well as in multiplicity distributions in a given rapidity window. The hadron abundances may reflect directly the chemical composition in the plasma phase.

- To identify the phase transition threshold the measurements should be done at different collision energies. The predicted dependence on the expansion rate and the reaction geometry can be checked in collisions with different ion masses and impact parameters.

- If the first order deconfinement/chiral phase transition is only possible at finite baryon densities, one should try to identify it by searching for the anomalous fluctuations in the regions of phase space characterized by a large baryon chemical potential. These could be the nuclear fragmentation regions in collisons with very high energies (highenergy SPS, RHIC, LHC) or the central rapidity region (AGS, low-energy SPS, future GSI facility FAIR).

The author is grateful to L.M. Satarov, M.I. Gorenstein and M. Gazdzicki for many fruitful discussions. This work was supported partly by the RFBR grant 05-02-04013 (Russia).

\section{References}

[1] M.A. Halasz, A.D. Jackson, R.E. Shrock, M.A. Stephanov and J.J.M. Verbarshot, Phys. Rev. D58, 096007 (1998).

[2] J. Berges and K. Rajagopal, Nucl. Phys. B538, 215 (1999).

[3] O. Scavenius, A. Mocsy, I.N. Mishustin, D. Rischke, Phys. Rev. C 64, 045202 (2001).

[4] M. Stephanov, K. Rajagopal and E. Shuryak, Phys. Rev. Lett. 81, 4816 (1998).

[5] Boris Berdnikov and Krishna Rajagopal, Phys. Rev. D61, 105017 (2000).

[6] I.N. Mishustin, Phys. Rev. Lett. 82, 4779 (1999).

[7] I.N. Mishustin, L.M. Satarov, H. Stoecker and W. Greiner, Phys. Rev. C59, 3243 (1999).

[8] W. Reisdorf and FOPI Collaboration, Nucl. Phys. A612, 493 (1997).

[9] Nu Xu, Prog. Part. Nucl. Phys. 53, 165 (2004).

[10] I.N. Mishustin, O. Scavenius, Phys. Rev. Lett. 83, 3134 (1999).

[11] O. Scavenius, A. Dumitru, E. S. Fraga, J. T. Lenaghan and A. D. Jackson, Phys. Rev. D 63, 116003 (2001).

[12] K. Paech, H. Stoecker and A. Dumitru, Phys. Rev. C 68, 044907 (2003).

[13] L.P. Csernai, I.N. Mishustin and A. Mocsy, Heavy Ion Phys., 3, 151 (1996);

A. Mocsy, M.Sc. thesis, University of Bergen, 1996.

[14] L.P. Csernai, I.N. Mishustin, Phys. Rev. Lett. 74, 5005 (1995).

[15] L.P. Csernai, J.I. Kapusta, Phys. Rev. Lett. 69, 737 (1992); Phys. Rev. D46, 1379 (1992).

[16] D.E. Grady, J. Appl. Phys. 53(1), 322 (1981). 
[17] B.L. Holian and D.E. Grady, Phys. Rev. Lett. 60, 1355 (1988).

[18] J. Bjorken, Phys. Rev. D27, 140 (1983)

[19] C.M. Huang and E.V. Shuryak, Phys. Rev. Lett. 75, 4003 (1995); Phys. Rev. C57, 1891 (1998).

[20] D. Rischke, M. Gyulassy, Nucl. Phys. A597, 701 (1996); A608, 479 (1996).

[21] I.N. Mishustin, Nucl. Phys. A630, 111c (1998).

[23] S.I. Digal, A.M Mapusta, Privastava Phys. Rev. D23, 2444 (1981). 80, 1841 (1998).

[24] M. Alford, K. Rajagopal and F. Wilczek, Phys. Lett. B422, 247 (1998).

[25] P. Braun-Münzinger, D. Magestro, K. Redlich, and J. Stachel, Phys. Lett. B518, 41 (2001);

P. Braun-Münzinger, J. Stachel, J. Phys. G: Part. Nucl. Phys. 28, 1971 (2002).

[26] G. Baym, H. Heiselberg, Phys. Lett. B469, 7 (1999).

[27] T. Abbot and E-802 Collaboration, Phys. Rev. C52, 2663 (1995).

[28] M. Rybczynski and NA49 Collaboration, J. Phys. Conf. Ser. 5, 74 (2005).

[29] V.P. Konchakovski, S. Haussler, M.I. Gorenstein, E.L. Bratkovskaya, M. Bleicher, and H. Stöcker, nucl-th/0511083.

[30] P. Currurthers andC.C. Shih, Phys. Lett. 127B, 242 (1983).

[31] Z. Koba, H.B. Nielson and P. Olesen, Nucl. Phys. B40, 317 (1972). 